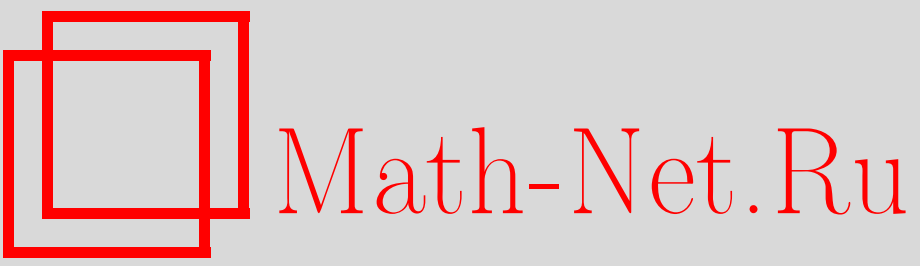

В. В. Грибанов, В. Г. Кадышевский, А. С. Сорин, Гамильтоновы структуры фермионных двумерных решеточных иерархий Тоды, ТМФ, 2006, том 146, номep 1, 90-102

DOI: https://doi.org/10.4213/tmf2011

Использование Общероссийского математического портала Math-Net.Ru подразумевает, что вы прочитали и согласны с пользовательским соглашением

http://www. mathnet.ru/rus/agreement

Параметры загрузки:

IP: 18.209 .158 .208

26 апреля 2023 г., 04:41:19 
ТЕОРЕТИЧЕСКАЯ

И МАТЕМАТИЧЕСКАЯ

ФИЗИКА

Том 146, № 1

январь, 2006

(c) 2006 г. В. В. Грибанов*, В. Г. Кадышевский*, А. С. Сорин*

\section{ГАМИЛЬТОНОВЫ СТРУКТУРЫ ФЕРМИОННЫХ ДВУМЕРНЫХ РЕШЕТОЧНЫХ ИЕРАРХИЙ ТОДЫ}

На базе соответствующих представлений Лакса предложен широкий класс интегрируемых двумерных фермионных решеточных иерархий Тоды, который включает двумерные $N=(2 \mid 2)$ и $N=(0 \mid 2)$ суперсимметричные решеточные иерархии Тоды как частные случаи. Развит обобщенный градуированный $R$-матричный формализм с использованием обобщенной градуированной скобки на пространстве градуированных операторов с инволюцией, обобщающей градуированный коммутатор в супералгебрах, что позволило описать указанные иерархии в рамках гамильтонова формализма и построить их первые две гамильтоновы структуры. Первая гамильтонова структура получена как для бозонных, так и для фермионных операторов Лакса, в то время как вторая гамильтонова структура найдена только для бозонных операторов Лакса.

Ключевые слова: интегрируемые системы, решетки Тоды, $R$-матрица, уравнение Янга-Бакстера.

\section{1. ВВЕДЕНИЕ}

Двумерная решеточная иерархия Тоды (РТ-иерархия) впервые была исследована в работах [1], и в настоящее время известны два различных нетривиальных суперсимметричных расширения двумерной РТ-иерархии. Эти расширения суть $N=(2 \mid 2)$ [2]-[7] и $N=(0 \mid 2)$ [7], [4] суперсимметричные РТ-иерархии, которые обладают разным числом суперсимметрий и содержат $N=(2 \mid 2)$ и $N=(0 \mid 2)$ РТуравнения в качестве подсистем. В последнее время были предложены двумерные обобщенные фермионные РТ-уравнения [6] и были рассмотрены две редукции этих уравнений, ведущие к $N=(2 \mid 2)$ и $N=(0 \mid 2)$ суперсимметричным РТ-уравнениям. $\mathrm{B}$ настоящей работе мы описываем широкий класс интегрируемых двумерных фермионных РТ-иерархий, который включает двумерные $N=(2 \mid 2)$ и $N=(0 \mid 2)$ суперсимметричные РТ-иерархии как частные случаи и содержит двумерные обобщенные фермионные РТ-уравнения в качестве подсистемы.

*Лаборатория теоретической физики им. Н.Н. Боголюбова, Объединенный институт ядерных исследований, Дубна, Московская обл., Россия. E-mail: gribanov@thsun1.jinr.ru, kadyshev@jinr.ru, sorin@thsun1.jinr.ru 
Совсем недавно в работе [8] было найдено гамильтоново описание двумерной РТиерархии в рамках $R$-матричного подхода, где ключевым моментом явилось введение новой $R$-матрицы, ассоциированной с расщеплением алгебры, заданной парой разностных операторов. В настоящей работе мы применим эту $R$-матрицу для пространства разностных $Z_{2}$-градуированных операторов и получим бигамильтонову структуру двумерной фермионной $(K, M)$-РТ-иерархии.

Примечательно, что при решении этой задачи обобщенная градуированная скобка (7) (см. ниже) на пространстве градуированных операторов с инволюцией находит новое применение. Данная скобка была введена в работе [9], где было показано, что $N=(1 \mid 1)$ суперсимметричная двумерная РТ-иерархия обладает естественным представлением Лакса в терминах именно такой скобки, что позволило построить бездисперсионную $N=(1 \mid 1)$ двумерную РТ-иерархию и ее представление Лакса. В настоящей работе мы используем обобщенную градуированную скобку для описания двумерной фермионной $(K, M)$-РТ-иерархии и нахождения двух ее гамильтоновых структур.

Работа организована следующим образом. В разделе 2 дано определение пространства $Z_{2}$-градуированных разностных операторов с инволюцией и приведены определение и свойства обобщенной градуированной скобки [9]. В разделе 3 даны теоретические основы $R$-матричного подхода, обобщенного на случай $Z_{2}$ градуированных разностных операторов. Мы задаем $R$-матрицу на ассоциативной алгебре g $Z_{2}$-градуированных разностных операторов, получаем градуированное модифицированное уравнение Янга-Бакстера и, используя обобщенную градуированную скобку, определяем две скобки Пуассона для функционалов на $\mathrm{g}^{\dagger}=\mathrm{g}$. Используя эти скобки Пуассона, можно задать гамильтоновы уравнения, которые эквивалентным образом переписываются в терминах представления Лакса. Основные результаты раздела 3 сформулированы в виде теоремы. В разделе 4 предложен широкий класс двумерных фермионных $(K, M)$-РТ-иерархий в терминах представления Лакса с обобщенной градуированной скобкой и построена алгебра их потоков. В разделе 5 результаты раздела 3 применяются для получения явных выражений двух гамильтоновых структур двумерной фермионной $(K, M)$-РТ-иерархии. В разделе 6 кратко сформулированы основные полученные результаты и перечислены проблемы, оставшиеся нерешенными.

\section{2. ПРОСТРАНСТВО РАЗНОСТНЫХ ОПЕРАТОРОВ}

В этом разделе мы определим важное для дальнейшего рассмотрения пространство разностных операторов. Эти операторы могут быть представлены в следующем общем виде:

$$
\mathbb{O}_{m}=\sum_{k=-\infty}^{\infty} f_{k, j}^{(m)} e^{(k-m) \partial}, \quad m, j \in \mathbb{Z} .
$$

Функции $f_{2 k, j}^{(m)}\left(f_{2 k+1, j}^{(m)}\right)$, параметризующие операторы, являются $Z_{2}$-градуированными бозонными (фермионными) полями на решетке с индексом решетки $j(j \in \mathbb{Z})$ 
и грассмановой четностью, определяемой индексом $k$ :

$$
d_{f_{k, j}^{(m)}}=|k| \bmod 2
$$

Везде ниже предполагается, что функции $f_{k, j}^{(m)}$ удовлетворяют нулевым граничным условиям на бесконечности

$$
\lim _{j \rightarrow \pm \infty} f_{k, j}^{(m)}=0
$$

Оператор $e^{k \partial}$ есть оператор сдвига, действие которого на решеточные поля заключается в дискретном сдвиге индекса решетки

$$
e^{l \partial} f_{k, j}^{(m)}=f_{k, j+l}^{(m)} e^{l \partial}
$$

Оператор сдвига обладает $Z_{2}$-четностью, определенной как

$$
d_{e^{l \partial}}^{\prime}=|l| \bmod 2 .
$$

Операторы $\mathbb{O}_{m}(1)$ допускают задание диагональной $Z_{2}$-четности

$$
d_{\mathbb{O}_{m}}=d_{f_{k, j}^{(m)}}+d_{e^{(k-m) \partial}}^{\prime}=|m| \bmod 2
$$

и инволюции

$$
\mathbb{O}_{m}^{*}=\sum_{k=-\infty}^{\infty}(-1)^{k} f_{k, j}^{(m)} e^{(k-m) \partial}
$$

Далее нам также потребуются проекторы операторов $\mathbb{O}_{m}$, определенные как

$$
\left(\mathbb{O}_{m}\right)_{\leqslant p}=\sum_{k \leqslant p+m} f_{k, j} e^{(k-m) \partial}, \quad\left(\mathbb{O}_{m}\right)_{\geqslant p}=\sum_{k \geqslant p+m} f_{k, j} e^{(k-m) \partial},
$$

и мы будем использовать обычные обозначения для проекторов $\left(\mathbb{O}_{m}\right)_{+}:=\left(\mathbb{O}_{m}\right)_{\geqslant 0}$ и $\left(\mathbb{O}_{m}\right)_{-}:=\left(\mathbb{O}_{m}\right)_{<0}$. Заметим, что $e^{l \partial}$ - это общепринятое обозначение для операторов сдвига, определенных в терминах бесконечномерных матриц $\left(e^{l \partial}\right)_{i, j} \equiv \delta_{i, j-l}$, и существует изоморфизм между операторами (1) и бесконечномерными матрицами (см., например, [10])

$$
\mathbb{O}_{m}=\sum_{k=-\infty}^{\infty} f_{k, j}^{(m)} e^{(k-m) \partial} \rightarrow\left(\mathbb{O}_{m}\right)_{j, i} \equiv \sum_{k=-\infty}^{\infty} f_{k, j}^{(m)} \delta_{j, i-k+m} .
$$

В пространстве операторов (1) можно выделить два подпространства, которые чрезвычайно важны в дальнейшем:

$$
\begin{array}{ll}
\mathbb{O}_{K_{1}}^{+}=\sum_{k=0}^{\infty} f_{k, j} e^{\left(K_{1}-k\right) \partial}, & K_{1} \in \mathbb{N}, \\
\mathbb{O}_{K_{2}}^{-}=\sum_{k=0}^{\infty} f_{k, j} e^{\left(k-K_{2}\right) \partial}, & K_{2} \in \mathbb{N} .
\end{array}
$$


Операторы подпространств $\mathbb{O}_{K_{1}}^{+}$и $\mathbb{O}_{K_{2}}^{-}$образуют ассоциативные алгебры с операцией умножения (3). Используя это обстоятельство, определим на этих подпространствах обобщенную градуированную алгебру со скобкой [9]

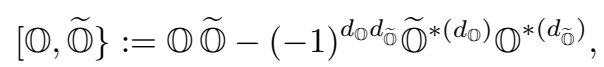

где операторы $\mathbb{O}$ и $\widetilde{\mathbb{O}}$ принадлежат подпространствам $\mathbb{O}_{K_{1}}^{+}$и $\mathbb{O}_{K_{2}}^{-}$, а $\mathbb{O}^{*(m)}$ обозначает $m$-кратное действие инволюции $*$ на оператор $\mathbb{O},\left(\mathbb{O}^{*(2)}=\mathbb{O}\right)$. Скобка $(7)$ обобщает (анти)коммутатор в супералгебрах и обладает следующими свойствами [9]:

симметрия

$$
[\mathbb{O}, \widetilde{\mathbb{O}}\}=-(-1)^{d_{0} d_{\widetilde{\Phi}}}\left[\widetilde{\mathbb{O}}^{*\left(d_{\oplus}\right)}, \mathbb{O}^{*\left(d_{\widetilde{\Phi}}\right)}\right\},
$$

дифференцирование

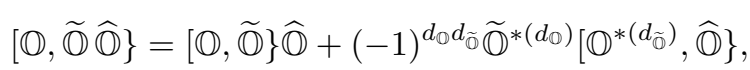

тождество Якоби

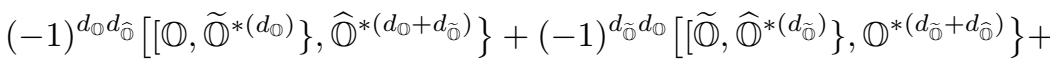

$$
\begin{aligned}
& +(-1)^{d_{\widehat{\Phi}} d_{\widetilde{\Phi}}}\left[\left[\widehat{\mathbb{O}}, \mathbb{O}^{*\left(d_{\widehat{\oplus}}\right)}\right\}, \widetilde{\mathbb{O}}^{*\left(d_{\widehat{\oplus}}+d_{\oplus}\right)}\right\}=0 .
\end{aligned}
$$

Для операторов $\mathbb{O}_{m}(1)$ определим также операцию взятия суперследа:

$$
\operatorname{str} \mathbb{O}=\sum_{j=-\infty}^{\infty}(-1)^{j} f_{m, j}^{(m)} .
$$

Нетрудно убедиться, что основное свойство суперследов $\operatorname{str}[\mathbb{O}, \widetilde{\mathbb{O}}\}=0$ в случае обобщенной градуированной скобки (7) действительно выполняется, если входящие в операторы (1) функции удовлетворяют нулевым граничным условиям на бесконечности (2).

\section{R-МАТРИЧНЫЙ ФОРМАЛИЗМ}

В этом разделе даются основы $R$-матричного подхода применительно к случаю пространства операторов (1).

Пусть g - некоторая ассоциативная алгебра операторов из пространства операторов (1) с инвариантным невырожденным скалярным произведением

$$
\langle\mathbb{O}, \widetilde{\mathbb{O}}\rangle=\operatorname{str}(\mathbb{O} \widetilde{\mathbb{O}}),
$$

используя которое можно отождествить алгебру g с дуальным к ней пространством $\mathrm{g}^{\dagger}$. Определим следующую скобку Пуассона:

$$
\{f, g\}(\mathbb{O})=-\left\langle\mathbb{O},\left[\nabla g,(\nabla f)^{*\left(\nabla d_{g}\right)}\right\}\right\rangle,
$$

где $f, g$ - функционалы на g, а $\nabla f$ и $\nabla g$ - их градиенты в точке $\mathbb{O}$, которые связаны с $f, g$ посредством скалярного произведения:

$$
\left.\frac{\partial f(\mathbb{O}+\epsilon \delta \mathbb{O})}{\partial \epsilon}\right|_{\epsilon=0}=\langle\delta \mathbb{O}, \nabla f(\mathbb{O})\rangle .
$$


Заметим, что надлежащие свойства скобки Пуассона (12) вытекают из свойств (8)$(10)$ обобщенной скобки $(7)$ и строго определяются $Z_{2}$-градуировкой оператора $\mathbb{O}$. Таким образом, мы имеем свойства симметрии

$$
\{f, g\}=-(-1)^{\left(d_{f}+d_{\emptyset}\right)\left(d_{g}+d_{0}\right)}\{g, f\},
$$

дифференцирования

$$
\{f, g h\}=\{f, g\} h+(-1)^{d_{g}\left(d_{f}+d_{\odot}\right)} g\{f, h\}
$$

и тождество Якоби

$$
\begin{gathered}
(-1)^{\left(d_{f}+d_{\odot}\right)\left(d_{h}+d_{\odot}\right)}\{\{f, g\}, h\}+(-1)^{\left(d_{g}+d_{\odot}\right)\left(d_{f}+d_{\odot}\right)}\{\{g, h\}, f\}+ \\
+(-1)^{\left(d_{h}+d_{\odot}\right)\left(d_{g}+d_{\odot}\right)}\{\{h, f\}, g\}=0 .
\end{gathered}
$$

Так, для оператора $\mathbb{O}$ с четной диагональной $Z_{2}$-градуировкой мы имеем обычную (четную) $Z_{2}$-градуированную скобку Пуассона, в то время как для операторов с нечетной диагональной градуировкой $d_{\mathbb{O}}(4)$ формула $(12)$ определяет нечетную $Z_{2}$ градуированную скобку Пуассона (антискобку).

Определив скобку Пуассона, перейдем к построению иерархии потоков, порождаемых этой скобкой через гамильтонианы. Следовательно, нам нужно определить бесконечное множество функционалов, которые, для того чтобы претендовать на роль гамильтонианов, должны быть в инволюции. Для скобки Пуассона (12) бесконечное множество гамильтонианов может быть найдено достаточно обычным способом:

$$
H_{k}=\frac{1}{k} \operatorname{str} \mathbb{O}_{*}^{k}=\frac{1}{k} \sum_{i=-\infty}^{\infty}(-1)^{i} f_{k m, i}^{(k m)},
$$

где операторы $\mathbb{O}_{*}^{k}$ определены следующим образом:

$$
(\mathbb{O})_{*}^{2 k}:=\left(\mathbb{O}^{*\left(d_{0}\right)} \mathbb{O}\right)^{k}, \quad(\mathbb{O})_{*}^{2 k+1}:=\mathbb{O}(\mathbb{O})_{*}^{2 k}
$$

Для нечетных операторов $\mathbb{O}$ выражение (16) определяет только фермионные ненулевые функционалы $H_{2 k+1}$, поскольку в этом случае четные степени операторов $\mathbb{O}$ имеют следующее представление:

$$
d_{\mathbb{O}}=1: \quad(\mathbb{O})_{*}^{2 k}=\left(\frac{1}{2}\left[(\mathbb{O})^{*}, \mathbb{O}\right\}\right)^{k} \equiv \frac{1}{2}\left[\left((\mathbb{O})_{*}^{2 k-1}\right)^{*}, \mathbb{O}\right\},
$$

и все бозонные гамильтонианы тривиальны $\left(H_{2 k}=0\right)$ вследствие равенства нулю суперследа обобщенной градуированной скобки.

Функционалы (16), очевидно, находятся в инволюции, однако порождаемая ими динамика тривиальна. Действительно, функционалы $H_{k}(16)$ суть операторы Казимира скобки Пуассона (12), т.е. скобка Пуассона функционала $H_{k}$ с любым другим функционалом равна нулю (в силу соотношения $\nabla H_{k+1}=\mathbb{O}_{*}^{k}$ ). Тем не менее возможно модифицировать скобку Пуассона (12) таким образом, чтобы новая скобка Пуассона порождала нетривиальные уравнения движения при использовании тех же 
гамильтонианов (16), а сами гамильтонианы по-прежнему находились в инволюции по отношению к модифицированной скобке Пуассона. Введем модифицированную обобщенную градуированную скобку на пространстве операторов (1)

$$
[\mathbb{O}, \widetilde{\mathbb{O}}\}_{R}:=[R(\mathbb{O}), \widetilde{\mathbb{O}}\}+[\mathbb{O}, R(\widetilde{\mathbb{O}})\},
$$

где оператор $R$ есть линейное отображение $R: \mathrm{g} \rightarrow \mathrm{g}$ такое, что скобка (19) обладает свойствами (8)-(10). Можно убедиться, что тождества Якоби (10) для скобки (19) могут быть эквивалентным образом переписаны в терминах обобщенной градуированной скобки (7):

$$
\begin{aligned}
& \left.\left.(-1)^{d_{\mathbb{O}} d_{\widehat{\mathbb{O}}}\left[\left[\mathbb{O}, \widetilde{\mathbb{O}}^{*}\left(d_{\mathbb{O}}\right)\right.\right.}\right\}_{R}, \widehat{\mathbb{O}}^{*\left(d_{\mathbb{O}}+d_{\widetilde{\mathbb{O}}}\right)}\right\}_{R}+\text { cycle perm. }= \\
& \quad=(-1)^{d_{\mathbb{O}} d_{\widehat{\mathbb{O}}}}\left[R\left(\left[\mathbb{O}, \widetilde{\mathbb{O}}^{*\left(d_{\mathbb{O}}\right)}\right\}_{R}\right)-\left[R(\mathbb{O}), R\left(\widetilde{\mathbb{O}}^{*\left(d_{\mathbb{O}}\right)}\right)\right\}, \widehat{\mathbb{O}}^{*\left(d_{\mathbb{O}}+d_{\widetilde{\mathbb{O}}}\right)}\right\}+\text { cycle perm. }=0 .
\end{aligned}
$$

Таким образом, мы приходим к заключению, что достаточным условием того, что отображение $R$ есть $R$-матрица, является выполнение следующего равенства:

$$
R\left([\mathbb{O}, \widetilde{\mathbb{O}}\}_{R}\right)-[R(\mathbb{O}), R(\widetilde{\mathbb{O}})\}=\alpha[\mathbb{O}, \widetilde{\mathbb{O}}\}
$$

где $\alpha$ - некоторая константа. Следуя принятой в [11] терминологии, мы назовем уравнение (20) градуированным модифицированным уравнением Янга-Бакстера. Уравнение (20) представляет собой обобщение градуированного модифицированного классического уравнения Янга-Бакстера, рассмотренного в работе [12], на пространство градуированных операторов (1).

Используя новую скобку (19), можно определить соответствующую новую скобку Пуассона на дуальном пространстве $\mathrm{g}^{\dagger}$ :

$$
\{f, g\}_{1}(\mathbb{O})=-\frac{1}{2}\left\langle\mathbb{O},\left[\nabla g,(\nabla f)^{*\left(d_{\nabla g}\right)}\right\}_{R}\right\rangle .
$$

Относительно зависимости правой части (21) от точки (O) эта скобка является линейной. Не вдаваясь в детали, введем также билинейную скобку для бозонных градуированных операторов $\mathbb{O}_{\mathrm{B}}\left(d_{\mathbb{O}_{\mathrm{B}}}=0\right)$ следующим образом:

$$
\begin{aligned}
\{f, g\}_{2}\left(\mathbb{O}_{\mathrm{B}}\right)=- & \frac{1}{4}\left\langle\left[\mathbb{O}_{\mathrm{B}}, \nabla g\right\} R\left((\nabla f)^{*\left(d_{\nabla g}\right)} \mathbb{O}_{\mathrm{B}}^{*\left(d_{\nabla f}+d_{\nabla g}\right)}+\mathbb{O}_{\mathrm{B}}^{*\left(d_{\nabla g}\right)}(\nabla f)^{*\left(d_{\nabla g}\right)}\right)-\right. \\
& \left.-R\left(\nabla g \mathbb{O}_{\mathrm{B}}^{*\left(d_{\nabla g}\right)}+\mathbb{O}_{\mathrm{B}} \nabla g\right)\left[\mathbb{O}_{\mathrm{B}}^{*\left(d_{\nabla g}\right)},(\nabla f)^{*\left(d_{\nabla g}\right)}\right\}\right\rangle .
\end{aligned}
$$

Билинейную скобку для случая фермионных операторов $\mathbb{O}_{\mathrm{F}}\left(d_{\mathbb{O}_{\mathrm{F}}}=1\right)$ нам построить не удалось. Скобка (21), очевидно, является скобкой Пуассона, если $R$ является $R$-матрицей на g. Билинейная скобка (22) становится скобкой Пуассона при более жестких условиях, которые даны в следующей теореме.

ТеоремА. А. Линейная скобка (21) является скобкой Пуассона, если $R$ удовлетворяет градуированному модифицированному уравнению Янга-Бакстера (20).

Б. Билинейная скобка (22) является скобкой Пуассона, если $R$ и ее антисимметричная часть $\left(R-R^{\dagger}\right) / 2$ удовлетворяют градуированному модифицированному 
уравнению Янга-Бакстера (20) с той же константой $\alpha$, где сопряженный оператор $R^{\dagger}$ действует на дуальном пространстве $\mathrm{g}^{\dagger}$ :

$$
\langle\mathbb{O}, R(\widetilde{\mathbb{O}})\rangle=\left\langle R^{\dagger}(\mathbb{O}), \widetilde{\mathbb{O}}\right\rangle .
$$

В. Если $\mathbb{O}=\mathbb{O}_{\mathrm{B}}$, то эти две скобки Пуассона совместны:

$$
\{f, g\}_{2}\left(\mathbb{O}_{\mathrm{B}}+b\right)=\{f, g\}_{2}\left(\mathbb{O}_{\mathrm{B}}\right)+b\{f, g\}_{1}\left(\mathbb{O}_{\mathrm{B}}\right) .
$$

Г. Операторы Казимира $H_{k}$ (16) скобки (12) находятся в инволюиии относительно как линейной (21), так и билинейной (22) скобок Пуассона.

Д. Гамильтонианы $H_{k} \neq 0$ (16) порождают уравнения движения

$$
\begin{aligned}
\partial_{k} \mathbb{O} & =\left\{H_{k+1}, \mathbb{O}\right\}_{1}=\frac{1}{2}\left[R\left(\left(\nabla H_{k+1}\right)^{*\left(d_{\mathbb{O}}\right)}\right), \mathbb{O}\right\}, \\
\partial_{k} \mathbb{O}_{\mathrm{B}} & =\left\{H_{k}, \mathbb{O}_{\mathrm{B}}\right\}_{2}=\frac{1}{4}\left[R\left(\nabla H_{k} \mathbb{O}_{\mathrm{B}}+\mathbb{O}_{\mathrm{B}} \nabla H_{k}\right), \mathbb{O}_{\mathrm{B}}\right\}
\end{aligned}
$$

посредством скобок (21) и (22), соответственно; эти уравнения связывают представления Лакса с гамильтоновым представлением.

Заметим, что аналогичная теорема для случая, когда операторы сдвига и функции, параметризующие разностные операторы $\mathbb{O}(1)$, имеют четную $Z_{2}$-градуировку, рассматривалась в работах [8], [11], [13].

\section{4. ДВУМЕРНАЯ ФЕРМИОННАЯ $(K, M)$-РТ-ИЕРАРХИЯ}

В этом разделе будет дана формулировка двумерной фермионной $(K, M)$-РТиерархии в терминах представления Лакса.

Рассмотрим два разностных оператора

$$
L_{K}^{+}=\sum_{k=0}^{\infty} u_{k, i} e^{(K-k) \partial}, \quad L_{M}^{-}=\sum_{k=0}^{\infty} v_{k, i} e^{(k-M) \partial},
$$

которые, очевидно, принадлежат соответственно пространствам (5) и (6). Поля на решетке и оператор сдвига, входящие в эти операторы, имеют следующие размерности в единицах длины: $\left[u_{k, i}\right]=-k / 2,\left[v_{k, i}\right]=(k-K-M) / 2$ и $\left[e^{k \partial}\right]=-k / 2$, так что операторы (23) имеют одинаковую размерность $\left[L_{K}^{+}\right]=\left[L_{M}^{-}\right]=-K / 2$. Динамика полей $u_{k, i}, v_{k, i}$ задается уравнениями Лакса, выраженными через обобщенную скобку (7) $[9]$ :

$$
\begin{gathered}
D_{s}^{ \pm} L_{\Omega^{\alpha}}^{\alpha}=\mp \alpha(-1)^{s \Omega^{\alpha} \Omega^{ \pm}}\left[\left(\left(\left(L_{\Omega^{ \pm}}^{ \pm}\right)_{*}^{s}\right)_{-\alpha}\right)^{*\left(\Omega^{\alpha}\right)}, L_{\Omega^{\alpha}}^{\alpha}\right\}, \\
\alpha=+,-, \quad \Omega^{+}=K, \quad \Omega^{-}=M, \quad s \in \mathbb{N},
\end{gathered}
$$

где $D_{s}^{ \pm}$- эволюционные производные, имеющие следующую $Z_{2}$-градуировку:

$$
d_{D_{s}^{+}}=s K \quad \bmod 2, \quad d_{D_{s}^{-}}=s M \quad \bmod 2,
$$


и размерность $\left[D_{s}^{+}\right]=\left[D_{s}^{-}\right]=-s K / 2$. Уравнения Лакса $(24)$ порождают неабелеву (супер)алгебру потоков двумерной фермионной $(K, M)$-РТ-иерархии

$$
\begin{gathered}
{\left[D_{s}^{+}, D_{p}^{+}\right\}=\left(1-(-1)^{s p K}\right) D_{s+p}^{+},} \\
{\left[D_{s}^{-}, D_{p}^{-}\right\}=\left(1-(-1)^{s p M}\right) D_{s+p}^{-}, \quad\left[D_{s}^{+}, D_{p}^{-}\right\}=0}
\end{gathered}
$$

Составные операторы $\left(L_{K}^{+}\right)_{*}^{s}$ и $\left(L_{M}^{-}\right)_{*}^{s}$, входящие в уравнения Лакса $(24)$, определены формулой (17) и также принадлежат соответственно пространствам (5) и (6),

$$
\left(L_{K}^{+}\right)_{*}^{r}:=\sum_{k=0}^{\infty} u_{k, i}^{(r)} e^{(r K-k) \partial}, \quad\left(L_{M}^{-}\right)_{*}^{r}:=\sum_{k=0}^{\infty} v_{k, i}^{(r)} e^{(k-r M) \partial} .
$$

Здесь $u_{k, i}^{(r)}$ и $v_{k, i}^{(r)}$ суть функционалы исходных полей, и для них имеют место следующие рекуррентные соотношения:

$$
\begin{array}{ll}
u_{p, i}^{(r+1)}=\sum_{k=0}^{p}(-1)^{k K} u_{k, i}^{(r)} u_{p-k, i-k+r K}, & u_{p, i}^{(1)}=u_{p, i}, \\
v_{p, i}^{(r+1)}=\sum_{k=0}^{p}(-1)^{k M} v_{k, i}^{(r)} v_{p-k, i+k-r M}, & v_{p, i}^{(1)}=v_{p, i} .
\end{array}
$$

Теперь, используя представление Лакса (24) и соотношения (9) и (17), можно получить уравнения движения для составных операторов Лакса

$$
\left.D_{s}^{ \pm}\left(L_{\Omega^{\alpha}}^{\alpha}\right)_{*}^{r}=\mp \alpha(-1)^{s r \Omega^{\alpha} \Omega^{ \pm}}\left[\left(\left(L_{\Omega^{ \pm}}^{ \pm}\right)_{*}^{s}\right)_{-\alpha}\right)^{*\left(r \Omega^{\alpha}\right)},\left(L_{\Omega^{\alpha}}^{\alpha}\right)_{*}^{r}\right\} .
$$

Представление Лакса (25) порождает следующие уравнения для функционалов $u_{k, i}^{(r)}$, $v_{k, i}^{(r)}$ :

$$
\begin{aligned}
D_{s}^{+} u_{k, i}^{(r)}= & \sum_{p=1}^{k}\left((-1)^{r p K+1} u_{p+s K, i}^{(s)} u_{k-p, i-p}^{(r)}+(-1)^{(k+p) s K} u_{k-p, i}^{(r)} u_{p+s K, i+p-k+r K}^{(s)}\right) \\
D_{s}^{-} u_{k, i}^{(r)}= & \sum_{p=0}^{s M-1}\left((-1)^{(s M+p) r K} v_{p, i}^{(s)} u_{p+k-s M, i+p-s M}^{(r)}-\right. \\
& \left.\quad-(-1)^{(k+p+1) s M} u_{p+k-s M, i}^{(r)} v_{p, i-p-k+s M+r K}^{(s)}\right) \\
D_{s}^{+} v_{k, i}^{(r)}= & \sum_{p=0}^{s K}\left((-1)^{(s K+p) r M} u_{p, i}^{(s)} v_{p+k-s K, i-p+s K}^{(r)}-\right. \\
& \left.-(-1)^{(k+p+1) s K} v_{p+k-s K, i}^{(r)} u_{p, i+p+k-s K-r M}^{(s)}\right) \\
D_{s}^{-} v_{k, i}^{(r)}= & \sum_{p=0}^{k}\left((-1)^{r p M+1} v_{p+s M, i}^{(s)} v_{k-p, i+p}^{(r)}+(-1)^{(k+p) s M} v_{k-p, i}^{(r)} v_{p+s M, i+k-p-r M}^{(s)}\right) .
\end{aligned}
$$

Предполагается, что в правых частях уравнений $(26)$ все функционалы $u_{k, i}^{(r)} v_{k, i}^{(r)} \mathrm{c}$ $k<0$ должны быть положены равными нулю.

4 Теоретическая и математическая физика, т. 146, № 1, 2006 г. 
Можно показать, что все известные в настоящее время фермионные двумерные РТ-уравнения [3]-[7] могут быть получены из системы уравнений (26) в качестве подсистем при наложении дополнительных условий редукции. Мы называем уравнения (24) двумерной фермионной $(K, M)$-РТ-иерархией.

\section{5. БИГАМИЛЬТОНОВА СТРУКТУРА ДВУМЕРНОЙ ФЕРМИОННОЙ $(K, M)$-РТ-ИЕРАРХИИ}

В этом разделе мы применим $R$-матричный подход для построения бигамильтоновой структуры двумерной фермионной $(K, M)$-РТ-иерархии. Данная иерархия определяется двумя операторами Лакса (23), принадлежащими к пространствам операторов (5), (6). Следуя работе [8], мы рассмотрим ассоциативную алгебру на пространстве прямых сумм двух разностных операторов

$$
\mathrm{g}:=\mathbb{O}_{K_{1}}^{+} \oplus \mathbb{O}_{K_{2}}^{-}
$$

Однако, в отличие от случая чисто бозонной двумерной РТ-иерархии, разностные операторы в прямой сумме (27) могут обладать как противоположной, так и одинаковой диагональной $Z_{2}$-градуировкой. Оказывается, что скобка Пуассона может быть корректно определена лишь в последнем случае. Ниже мы ограничимся случаем, когда оба оператора в g (27) имеют одинаковую диагональную четность.

Обозначим через $\left(x^{+}, x^{-}\right)$элементы такой алгебры $\mathrm{g}=\mathrm{g}^{\dagger}$ с операцией умножения

$$
\left(x_{1}^{+}, x_{1}^{-}\right) \cdot\left(x_{2}^{+}, x_{2}^{-}\right)=\left(x_{1}^{+} x_{2}^{+}, x_{1}^{-} x_{2}^{-}\right)
$$

и определим скалярное произведение следующим образом:

$$
\left\langle\left(x^{+}, x^{-}\right)\right\rangle:=\operatorname{str}\left(x^{+}+x^{-}\right),
$$

где $x^{+} \in \mathbb{O}_{K_{1}}^{+}, x^{-} \in \mathbb{O}_{K_{2}}^{-}$. Используя это определение, зададим скобки Пуассона в виде

$$
\left\{f_{1}, f_{2}\right\}=\left\langle\left(\mathbb{O}_{K_{1}}^{+}, \mathbb{O}_{K_{2}}^{-}\right),\left[\nabla f_{1}, \nabla f_{2}\right\}^{\oplus}\right\rangle,
$$

где

$$
\left[\nabla f_{1}, \nabla f_{2}\right\}^{\oplus}:=\left(\left[\nabla f_{1}^{+},\left(\nabla f_{2}^{+}\right)^{*\left(d_{\nabla f_{1}^{+}}\right)}\right\},\left[\nabla f_{1}^{-},\left(\nabla f_{2}^{-}\right)^{*\left(d_{\nabla f_{1}^{-}}\right)}\right\}\right),
$$

$f_{k}$ - функционалы на g $(27)$ и $\nabla f_{k}\left[\left(\mathbb{O}_{K_{1}}^{+}, \mathbb{O}_{K_{2}}^{-}\right)\right]=\left(\nabla f_{k}^{+}, \nabla f_{k}^{-}\right)$- градиенты этих функционалов, которые могут быть найдены из определения

$$
\begin{aligned}
& \left.\frac{\partial f_{k}\left[\left(\mathbb{O}_{K_{1}}^{+}, \mathbb{O}_{K_{2}}^{-}\right)+\epsilon\left(\delta \mathbb{O}_{K_{1}}^{+}, \delta \mathbb{O}_{K_{2}}^{-}\right)\right]}{\partial \epsilon}\right|_{\epsilon=0}=\left\langle\left(\delta \mathbb{O}_{K_{1}}^{+}, \delta \mathbb{O}_{K_{2}}^{-}\right),\left(\nabla f_{k}^{+}, \nabla f_{k}^{-}\right)\right\rangle= \\
& =\left\langle\delta \mathbb{O}_{K_{1}}^{+}, \nabla f_{k}^{+}\right\rangle+\left\langle\delta \mathbb{O}_{K_{2}}^{-}, \nabla f_{k}^{-}\right\rangle .
\end{aligned}
$$

Для того чтобы получить нетривиальную гамильтонову динамику, нужно модифицировать скобки (30), используя $R$-матрицу:

$$
\left[\nabla f_{1}, \nabla f_{2}\right\}^{\oplus} \longrightarrow\left[\nabla f_{1}, \nabla f_{2}\right\}_{R}^{\oplus}=\left[R\left(\nabla f_{1}\right), \nabla f_{2}\right\}^{\oplus}+\left[\nabla f_{1}, R\left(\nabla f_{2}\right)\right\}^{\oplus} .
$$


$R$-матрица действует на пространстве (27) нетривиальным образом, перемешивая элементы двух подалгебр в прямой сумме между собой:

$$
R\left(x^{+}, x^{-}\right)=\left(x_{+}^{+}-x_{-}^{+}+2 x_{-}^{-}, x_{-}^{-}-x_{+}^{-}+2 x_{+}^{+}\right),
$$

что является ключевым моментом $R$-матричного подхода в двумерном случае [8]. Именно такая $R$-матрица позволяет найти две совместные пуассоновы структуры и переписать представление Лакса (24) в гамильтоновом виде. Действительно, данная $R$-матрица удовлетворяет градуированному модифицированному уравнению ЯнгаБакстера

$$
R\left(\left[\left(x^{+}, x^{-}\right),\left(y^{+}, y^{-}\right)\right\}_{R}\right)-\left[R\left(x^{+}, x^{-}\right), R\left(y^{+}, y^{-}\right)\right\}=\alpha\left[\left(x^{+}, x^{-}\right),\left(y^{+}, y^{-}\right)\right\}
$$

с $\alpha=1$. Прямая проверка подстановкой в (32) показывает, что антисимметричная часть

$$
\frac{1}{2}\left(R\left(x^{+}, x^{-}\right)-R^{\dagger}\left(x^{+}, x^{-}\right)\right)=\left(x_{>0}^{+}-x_{<0}^{+}-x_{0}^{-}, x_{<0}^{-}-x_{>0}^{-}+x_{0}^{+}\right)
$$

также удовлетворяет градуированному модифицированному уравнению Янга-Бакстера (32). Следовательно, в силу теоремы из раздела 3 на алгебре g (27) существуют две пуассоновы структуры.

Используя формулы $(21),(22),(28),(31)$ и циклические перестановки внутри суперследа (11), мы получаем следующий общий вид первой и второй скобок Пуассона:

$$
\{f, g\}_{i}=\left\langle P_{i}^{+}\left(\nabla g^{+}, \nabla g^{-}\right),\left(\nabla f^{+}\right)^{*\left(d_{\nabla g}\right)}\right\rangle+\left\langle P_{i}^{-}\left(\nabla g^{+}, \nabla g^{-}\right),\left(\nabla f^{-}\right)^{*\left(d_{\nabla g}\right)}\right\rangle,
$$

где $i=1,2$ и $d_{\nabla g}:=d_{\nabla g^{+}}=d_{\nabla g^{-}}$. Для первой гамильтоновой структуры явный вид тензора Пуассона в выражении (33) найден для любых значений $(K, M)$ :

$$
\begin{aligned}
& P_{1}^{+}\left(\nabla g^{+}, \nabla g^{-}\right)=\left[\left(\nabla g_{-}^{-}-\nabla g_{-}^{+}\right)^{*(K)},\left(L_{K}^{+}\right)^{*\left(d_{\nabla g}\right)}\right\}-\left(\left[L_{K}^{+}, \nabla g^{+}\right\}+\left[L_{M}^{-}, \nabla g^{-}\right\}\right)_{\leqslant 0}, \\
& P_{1}^{-}\left(\nabla g^{+}, \nabla g^{-}\right)=\left[\left(\nabla g_{+}^{+}-\nabla g_{+}^{-}\right)^{*(M)},\left(L_{M}^{-}\right)^{*\left(d_{\nabla g}\right)}\right\}-\left(\left[L_{K}^{+}, \nabla g^{+}\right\}+\left[L_{M}^{-}, \nabla g^{-}\right\}\right)_{>0},
\end{aligned}
$$

тогда как в случае второй гамильтоновой структуры формула (33) верна только для четных значений $(K, M)$ с тензором Пуассона

$$
\begin{aligned}
& P_{2}^{+}\left(\nabla g^{+}, \nabla g^{-}\right)= \\
& =\frac{1}{2}\left(\left[\left(\nabla g^{-}\left(L_{M}^{-}\right)^{*\left(d_{g}\right)}+L_{M}^{-} \nabla g^{-}-\nabla g^{+}\left(L_{K}^{+}\right)^{*\left(d_{g}\right)}-L_{K}^{+} \nabla g^{+}\right)_{-},\left(L_{K}^{+}\right)^{*\left(d_{g}\right)}\right\}-\right. \\
& \left.\quad-L_{K}^{+}\left(\left[L_{K}^{+}, \nabla g^{+}\right\}+\left[L_{M}^{-}, \nabla g^{-}\right\}\right)_{\leqslant 0}-\left(\left[L_{K}^{+}, \nabla g^{+}\right\}+\left[L_{M}^{-}, \nabla g^{-}\right\}\right)_{\leqslant 0}\left(L_{K}^{+}\right)^{*\left(d_{g}\right)}\right), \\
& P_{2}^{-}\left(\nabla g^{+}, \nabla g^{-}\right)= \\
& =\frac{1}{2}\left(\left[\left(\nabla g^{+}\left(L_{K}^{+}\right)^{*\left(d_{g}\right)}+L_{K}^{+} \nabla g^{+}-\nabla g^{-}\left(L_{M}^{-}\right)^{*\left(d_{g}\right)}-L_{M}^{-} \nabla g^{-}\right)_{+},\left(L_{M}^{-}\right)^{*\left(d_{g}\right)}\right\}-\right. \\
& \left.\quad-L_{M}^{-}\left(\left[L_{K}^{+}, \nabla g^{+}\right\}+\left[L_{M}^{-}, \nabla g^{-}\right\}\right)_{>0}-\left(\left[L_{K}^{+}, \nabla g^{+}\right\}+\left[L_{M}^{-}, \nabla g^{-}\right\}\right)_{>0}\left(L_{M}^{-}\right)^{*\left(d_{g}\right)}\right) .
\end{aligned}
$$

Скобки Пуассона для функций $u_{n, i}$ и $v_{n, i}$, параметризующих операторы Лакса (23), могут быть явно получены из (33), если принять во внимание, что

$$
\begin{aligned}
& \nabla u_{n, \xi} \equiv\left(\nabla u_{n, \xi}^{+}, \nabla u_{n, \xi}^{-}\right)=\left(e^{(n-K) \partial}(-1)^{i} \delta_{i, \xi}, 0\right), \\
& \nabla v_{n, \xi} \equiv\left(\nabla v_{n, \xi}^{+}, \nabla v_{n, \xi}^{-}\right)=\left(0, e^{(M-n) \partial}(-1)^{i} \delta_{i, \xi}\right) .
\end{aligned}
$$


Таким образом можно получить следующие выражения:

$$
\begin{array}{r}
\left\{u_{n, i}, u_{m, j}\right\}_{1}=(-1)^{j}\left(\delta_{n, K}^{-}+\delta_{m, K}^{-}-1\right)\left(u_{n+m-K, i} \delta_{i, j+n-K}-\right. \\
\left.-(-1)^{(m+K)(n+K+1)} u_{n+m-K, j} \delta_{i, j-m+K}\right), \\
\left\{u_{n, i}, v_{m, j}\right\}_{1}=(-1)^{j}\left[( \delta _ { n , K } ^ { - } - 1 ) \left(v_{m-n+K, i} \delta_{i, j+n-K}-\right.\right. \\
\left.-(-1)^{(m+M)(n+K+1)} v_{m-n+K, j} \delta_{i, j+m-M}\right)- \\
-\delta_{m, M}^{+}\left(u_{n-m+M, i} \delta_{i, j+n-K}-\right. \\
\left.\left.\quad-(-1)^{(m+M)(n+K+1)} u_{n-m+M, j} \delta_{i, j+m-M}\right)\right] \\
\left\{v_{n, i}, v_{m, j}\right\}_{1}=(-1)^{j}\left(1-\delta_{n, M}^{+}-\delta_{m, M}^{+}\right)\left(v_{n+m-M, i} \delta_{i, j-n+M}-\right. \\
\left.-(-1)^{(m+M)(n+M+1)} u_{n+m-M, j} \delta_{i, j+m-M}\right)
\end{array}
$$

для первой гамильтоновой структуры и

$$
\begin{aligned}
& \left\{u_{n, i}, u_{m, j}\right\}_{2}=-(-1)^{j} \frac{1}{2}\left[u_{n, i} u_{m, j}\left(\delta_{i, j+n-K}-(-1)^{m} \delta_{i, j-m+K}\right)+\sum_{k=0}^{n+m}\left(\delta_{m, k}^{+}-\delta_{m, k}^{-}\right) \times\right. \\
& \left.\quad \times\left((-1)^{m k} u_{n+m-k, i} u_{k, j} \delta_{i, j+n-k}-(-1)^{m(n+k+1)} u_{k, i} u_{n+m-k, j} \delta_{i, j-m+k}\right)\right], \\
& \left\{u_{n, i}, v_{m, j}\right\}_{2}=-(-1)^{j} \frac{1}{2}\left[u _ { n , i } v _ { m , j } \left(\delta_{i, j}+\delta_{i, j+n-K}-\right.\right. \\
& \left.\quad-(-1)^{m}\left(\delta_{i, j+m-M}+\delta_{i, j+n+m-K-M}\right)\right)+ \\
& \quad+2 \sum_{k=\max (0, m-n)}^{m-1}\left(u_{n-m+k, i} v_{k, j+m-k} \delta_{i, j+n-K}-\right. \\
& \left.\left.\quad-(-1)^{(n+1) m} v_{k, j} u_{n-m+k, i+k-m} \delta_{i, j+m+M}\right)\right], \\
& \left\{v_{n, i}, v_{m, j}\right\}_{2}=(-1)^{j} \frac{1}{2}\left[v_{n, i} v_{m, j}\left(\delta_{i, j-n+M}-(-1)^{m} \delta_{i, j+m-M}\right)-\right. \\
& \quad \sum_{k=0}^{n+m}\left(\delta_{m, k}^{+}-\delta_{m, k}^{-}\right)\left((-1)^{m k} v_{n+m-k, i} v_{k, j} \delta_{i, j-n+k}-\right. \\
& \left.\left.\quad-(-1)^{m(n+k+1)} v_{k, i} v_{n+m-k, j} \delta_{i, j+m-k}\right)\right]
\end{aligned}
$$

для второй гамильтоновой структуры, где

$$
\delta_{n, m}^{+}=\left\{\begin{array}{ll}
1, & n>m, \\
0, & n \leqslant m,
\end{array} \quad \delta_{n, m}^{-}= \begin{cases}1, & n<m \\
0, & n \geqslant m\end{cases}\right.
$$

Напомним, что вторая гамильтонова структура определена только для четных значений $(K, M)$.

Полученные гамильтоновы структуры обладают свойствами (13)-(15) с $d_{\mathbb{O}}=$ $d_{L_{K}^{+}}=d_{L_{M}^{-}}$. Используя эти свойства, можно переписать потоки иерархии $(26)$ для 
четных значений $(K, M)$ в бигамильтоновом виде

$$
D_{s}^{ \pm}\left(\begin{array}{c}
u_{n, i}^{(r)} \\
v_{n, i}^{(r)}
\end{array}\right)=\left\{\left(\begin{array}{c}
u_{n, i}^{(r)} \\
v_{n, i}^{(r)}
\end{array}\right), H_{s+1}^{ \pm}\right\}_{1}=\left\{\left(\begin{array}{c}
u_{n, i}^{(r)} \\
v_{n, i}^{(r)}
\end{array}\right), H_{s}^{ \pm}\right\}_{2},
$$

с гамильтонианами

$$
\begin{aligned}
& H_{s}^{+}=\frac{1}{s} \operatorname{str}\left(L_{K}^{+}\right)_{*}^{s}=\frac{1}{s} \sum_{i=-\infty}^{\infty}(-1)^{i} u_{s K, i}^{(s)}, \\
& H_{s}^{-}=\frac{1}{s} \operatorname{str}\left(L_{M}^{-}\right)_{*}^{s}=\frac{1}{s} \sum_{i=-\infty}^{\infty}(-1)^{i} v_{s M, i}^{(s)} .
\end{aligned}
$$

Для нечетных значений $(K, M)$ можно воспроизвести только бозонные потоки уравнений (26). В этом случае выражения (35) в силу соотношения (18) дают только фермионные ненулевые гамильтонианы, используя которые можно воспроизвести бозонные потоки посредством нечетной первой гамильтоновой структуры (34)

$$
D_{2 s}^{ \pm}\left(\begin{array}{c}
u_{n, i}^{(r)} \\
v_{n, i}^{(r)}
\end{array}\right)=\left\{\left(\begin{array}{c}
u_{n, i}^{(r)} \\
(r) \\
v_{n, i}^{(r)}
\end{array}\right), H_{2 s+1}^{ \pm}\right\}_{1}
$$

\section{6. ЗАКЛЮЧЕНИЕ}

В настоящей работе мы обобщили $R$-матричный подход на случай $Z_{2}$-градуированных операторов с инволюцией и показали, что на пространстве таких операторов существуют две пуассоновы структуры. Первая скобка Пуассона определена как для нечетных, так и для четных операторов с $Z_{2}$-градуировкой, тогда как вторая найдена только для четных операторов. Показано, что свойства скобок Пуассона обеспечиваются свойствами обобщенной градуированной скобки. Далее, мы предложили широкий класс двумерных фермионных $(K, M)$-РТ-иерархий в терминах представления Лакса с обобщенной градуированной скобкой и применили развитый $R$-матричный формализм для построения бигамильтоновых структур предложенных иерархий. Для четных значений $(K, M)$ получены как первая, так и вторая четные гамильтоновы структуры, и в этом случае все потоки двумерной фермионной $(K, M)$-РТ-иерархии могут быть переписаны в бигамильтоновом виде. Для нечетных значений $(K, M)$ найдена нечетная первая гамильтонова структура, и в этом случае только бозонные потоки двумерной фермионной $(K, M)$-РТ-иерархии могут быть представлены в гамильтоновом виде с использованием фермионных гамильтонианов.

Таким образом, проблема гамильтонова описания фермионных потоков двумерной фермионной $(K, M)$-РТ-иерархии осталась открытой. Другими задачами, все еще требующими ответа, являются построение второй гамильтоновой структуры (если она существует) для нечетных операторов Лакса и гамильтоновых структур (если они существуют) для операторов Лакса $L_{K}^{+}$и $L_{M}^{-}$с противоположными $Z_{2^{-}}$ градуировками. Не менее важным является вопрос о взаимосвязи между предложенным в настоящей работе градуированным модифицированным уравнением Янга- 
Бакстера (20) и градуированным классическим уравнением Янга-Бакстера, введенным в пионерской работе [14]. Все эти вопросы станут предметом дальнейших исследований.

Благодарности. Авторы благодарны А. П. Исаеву, П. П. Кулишу и А. А. Владимирову за полезные обсуждения. Данная работа была частично поддержана RFBRDFG (грант № 04-02-04002), DFG (грант № 436 RUS 113/669-2), грантом НАTO PST.GLG.980302 и Программой Гейзенберга-Ландау.

\section{Список литературы}

[1] А. В. Михайлов. Письма в ЖЭТФ. 1979. Т. 30. № 7. С. 443; K Ueno, K. Takasaki. Adv. Stud. Pure Math. 1984. V. 4. P. 1.

[2] M. A. Olshanetsky. Commun. Math. Phys. 1983. V. 88. P. 63; D. A. Leites, M. V. Saveliev, V.V. Serganova. Embeddings of $\operatorname{osp}(1 \mid 2)$ and the associated nonlinear supersymmetric equations. In: Group Theoretical Methods in Physics (Yurmala, 1985). V. I. Eds. M. A. Markov, V. I. Manko, V. V. Dodonov. Utrecht: VNU Sci. Press, 1986. Р. 255; В. А. Андреев. ТМФ. 1987. Т. 72. № 1. С. 112.

[3] K. Ikeda. Lett. Math. Phys. 1987. V. 14. P. 321; J. Evans, T. Hollowood. Nucl. Phys. B. 1991. V. 352. P. 723; O. Lechtenfeld, A. Sorin. Nucl. Phys. B. 1999. V. 557. P. 535; J. Nonlinear Math. Phys. 2001. V. 8. P. 183; 2004. V. 11. P. 294

[4] V. G. Kadyshevsky, A.S. Sorin. Supersymmetric Toda lattice hierarchies. In: Integrable Hierarchies and Modern Physical Theories. Eds. H. Aratyn, A. S. Sorin. Dordrecht-BostonLondon: Kluwer, 2001. P. 289; nlin.SI/0011009.

[5] V.V. Gribanov, V.G. Kadyshevsky, A.S. Sorin. Czech. J. Phys. 2004. V. 54. P. 1289.

[6] V. V. Gribanov, V. G. Kadyshevsky, A.S. Sorin. Discrete Dyn. Nat. Soc. 2004. V. 2004 № 1. P. 113.

[7] V. B. Derjagin, A. N. Leznov, A. Sorin. Nucl. Phys. B. 1998. V. 527. P. 643.

[8] G. Carlet. The Hamiltonian structures of the two-dimensional Toda lattice and R-matrices. math-ph/0403049.

[9] В. Г. Кадышевский, А. С. Сорин. ТМФ. 2002. Т. 132. № 2. С. 222; nlin.SI/0206044.

[10] H. Aratyn, K. Bering. Int. J. Mod. Phys. A. 2005. V. 20. P. 1367; nlin.SI/0402014.

[11] М. А. Семенов-Тян-Шанский. Функц. анализ и его прилож. 1983. Т. 17. С. 17.

[12] C. M. Yung. Mod. Phys. Lett. 1993. V. 8. P. 129.

[13] W. Oevel, O. Ragnisco. Physica A. 1989. V. 161. P. 181; L. Ch. Li, S. Parmenter. Commun. Math. Phys. 1989. V. 125. P. 545.

[14] П. П. Кулиш, Е. К. Склянин. Зап. научн. семин. ЛОМИ. 1980. Т. 95. С. 129. 